Rodrigo Pérez

Amable López

José Andrés Somolinos

Luis Ramón Núñez

http://dx.doi.org/10.21278/brod69103

ISSN 0007-215X

eISSN 1845-5859

\title{
DETAIL DESIGN OF A BALLAST CONTROL ROOM FOR AN UNDERWATER TIDAL ENERGY CONVERTER
}

UDC 629.5.584: 629.5.043: 629.5.062.2

Professional paper

\section{Summary}

The aim of this article is to design, precisely and fully detailed, the Control Room of a Ballast Actuation System of a marine Tidal Energy Converter. The design would respond to the detail design stage of any shipyard for its subsequent manufacture.

During the following lines, the authors have analysed the Ballast Actuation System, not only from a conceptual way, but also from the detailed design point of view, so it has been considered necessary to complete it, with a study of a technological nature, in which industrial (reducing the cost and increasing reliability) solutions have been developed for measuring and driving systems.

To elaborate the 3D detail design of the pump room, a topological design has been introduced for allowing a quicker evaluation of different design alternatives. All the machinery, piping, supports and auxiliary structures have been modelled in order to evaluate the quality of the conceptual and initial design. The primary structure, as well as the location of the equipment, have been carefully studied and analysed.

The P\&ID (Piping and Instrumentation Diagrams) and access drawings have been followed in order to model the 3D pipe routing, supports and auxiliary structure. The 3D components have been defined from its main features (element ID, supplier, description, weight and centre of gravity) and its geometry (library or geometric macro model), provided by the supplier. All this work has been carried out with the intention of not only assess different alternatives, but also to generate at any time the information for manufacturing and mounting.

Key words: $\quad$ Tidal Energy Converter, Pump Room, Ballast Systems

\section{Introduction}

This technical paper presents the most significant results of the study of a Ballast Actuation System (BAS), carried out by a Research and Development (R\&D) group of the Marine Engineering Technical School GIT-ERM from the Technical University of Madrid (ETSIN-UPM), consisting of the development of a specific and innovative Ballast Control 
System (BCS) enabling to avoid control problems associated with free surface ballast tanks [7] [9] [13].

For this analysis, it has been chosen a device that control depth and trim as the prototype for study. The device is a Tidal Energy Converter (TEC) with the following dimensions $11 \mathrm{x}$ $2 \times 12$ meters wingspan, with a rotor of 6.6 meters of diameter [6]. Within each float/hull has three tanks of ballast water and a Double Pump Type Ballast Actuation System (DPTBAS).

In operation, it means producing energy, this TEC works with the rotor shaft horizontal, entering the stream in the direction of the axis of the hulls, by the rounded part of them and keeping the operation depth, with a system of anchoring and mooring quite simple. Increasing the speed of the stream, the hydrodynamic forces will grow and in order to maintain the position, it is necessary to increase the net force of thrust, emptying the ballast water tank [4].

When the flow direction of the stream changes in each tidal cycle, it is necessary to turn the device $180^{\circ}$, passing through a vertical position with the rotor upwards [5]. To perform maintenance operations, it is necessary to emerge most of the device out of the water, eliminating the majority of the ballast water, always with the vertical axis perpendicular with the water surface [9].

Each float/hull is a separate drive system, but with a BCD integrated for the whole TEC. The system proposed, developed in this paper, is focused in elements, pipes, supports, auxiliary structures and circuits, but also it is presented an initial proposal of the sensors and control strategy.

\section{Manufacturing design of the Ballast Actuation System}

As these devices are not manned, and with a difficult access for maintenance, the complexity of manoeuvres in immersion (similar to a submarine) must be carried out in an autonomous way, efficient, accurate and with high reliability systems. After studying different alternatives [8], it has been perceived that a main driver for the selection of the BCS is the great volume of the tanks, plus the use of industrial elements adapted to the marine environment. After a complete study, the following main alternatives have been studied:

- Use of the pressure air for the water movement.

- Use of volumetric pumps.

- Use of centrifugal pumps.

As the water ballast needs to take water from the sea, this water is salty and with different solid particles, the use of spindle screw pumps or lobe pump is not feasible, so among the volumetric pump, flexible impeller pumps were chosen.

Starting from these conditions and of a preliminary specification, different BAS have been developed from a functional schema level. The selection process has been based on systems used in the maritime sector. Each one of these systems was revised by the R\&D group of the ETSIN-UPM GIT-ERM, analysing their advantages, disadvantages and proposing modifications to the development of a new alternative. As a consequence of this process, four alternatives have been analysed, and visualizing the results of the different solutions, it was designed a BAS as a solution used by a simple air system management with flexible impeller pumps for the precise control, centrifugal pumps for managing immersion/submersion manoeuvres.

This system, that it will be referred to as a BAS based on Double Pump Type (DPTBAS), is the one to be developed and design during the following paragraphs in this paper. 
After the analysis of the different solutions done above, it was concluded that the design principles of this system should be as follow:

- Insertion of man-holes in every tank, which it allows the incorporation of active elements working in its interior, to be possible its maintenance. According to this, air/water separators have been eliminated situating two independent pipes to each corner of the tank. Placing at the end of each pipe level binary sensors, which act as air/water detectors, producing that when the fluid is not the correct one on the pipe, an order is given to close the valve, located in the DPTBAS.

- Pumping hybrid solution, with three flexible impeller pumps for precision control manoeuvres in immersion and two centrifugal pumps for the immersion/submersion manoeuvres, scaled to manage that this types of movements could be achieved in the times indicated on the paper [1].

\section{Structure design of the Ballast Actuation System}

To develop the best possible design, it is mandatory to estimate all calculus that allow to use the maximum volume inside the DPTBAS.

When the structure of a cylindrical submarine is being designing, the first decision is to decide if the structure would have frames or not, and later on to calculate what would be the penalization about shell plate thickness if there are not frames.

After that it's important to define the steel thickness of a cylindrical tank, with main dimensions of four meters long and two meters of diameter, with the ability to submerge until a maximum operational depth of 25 meters, where the TEC device is optimized to obtain the maximum power, while the bottom is 50 meters from the surface.

In a previous design, with grade-A steel, with stiffeners (frames) and margin due to corrosion, the shell plating thickness was of $10(\mathrm{~mm})$.

In the present study is considered a hollow cylinder, closed at the ends by two watertight bulkheads, as appears in figure 1. For this preliminary study, it is considered a cylinder without frames that will be subjected to the pressure outside of the sea.

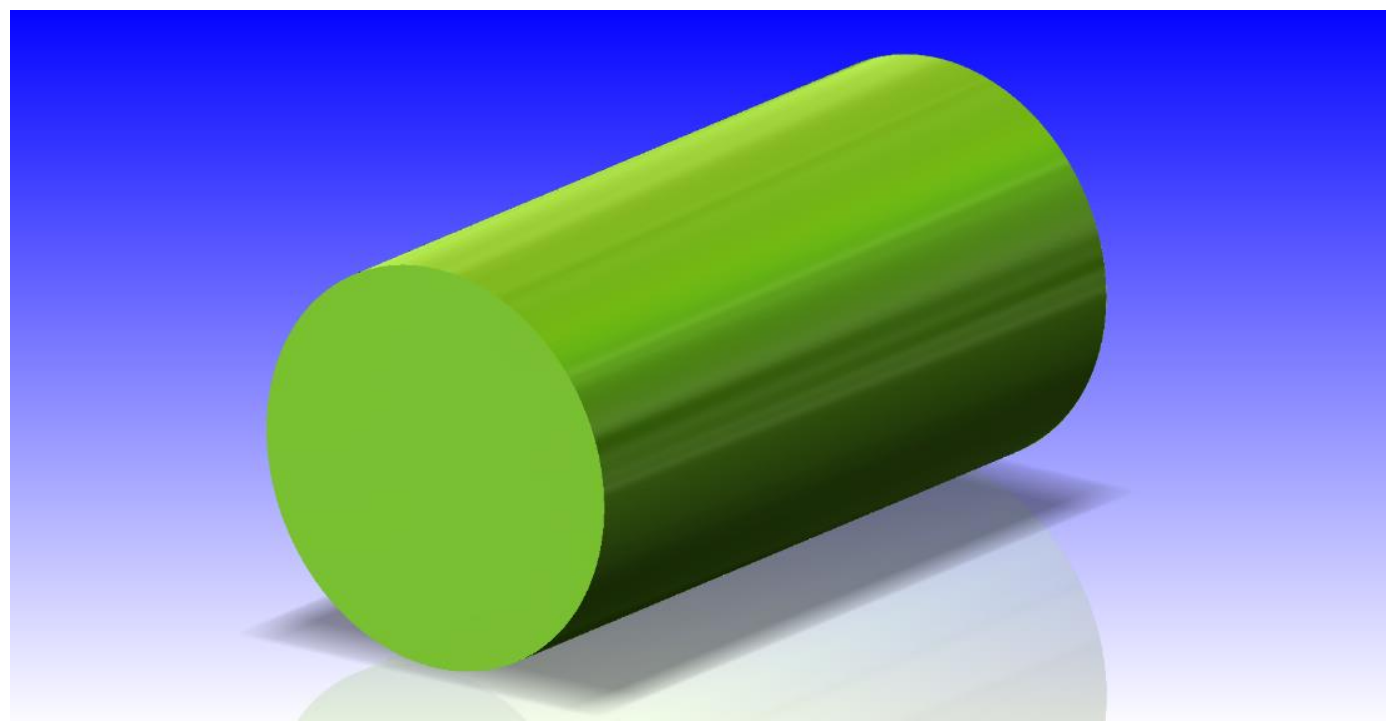

Fig. 1 Cylindrical tank to be analysed 
To calculate the hull pressure, the regulations of the classification society American Bureau of Shipping (ABS) have been followed [2]. It's necessary to calculate the maximum hull pressure according to different depths, based in the overpressure of the cylindrical hull, and of this way, find the shell thickness. This maximum pressure is the critical one for overpressure in the shell multiplied by a security factor, namely $\gamma$. The critical pressure can be also considered as the working pressure that exceeds the creep limit of the material, which produce the following equation (1):

$$
P_{y}(h)=\frac{\sigma_{y} h}{R(1-F)},
$$

Where $\boldsymbol{\sigma}_{\mathbf{y}}$ is the yield stress of the material limit, $\mathbf{h}$ is the shell thickness, and $\mathbf{R}$ is the radius of the cylinder. The value of $\mathbf{F}$ depends on material, hull cylinder forms and the frames that in the case of this structure non-existent. As it was mentioned above, the study aims to estimate the value of the thickness of the hull without reinforcements, so be used $\mathbf{F}=\mathbf{0}$ in the equation (1) for a cylindrical hull without frames.

ABS legislation compares this pressure $\mathbf{P}_{\mathbf{y}}$ with the buckling pressure of $\operatorname{Von} \operatorname{Misses}\left(\mathbf{P}_{\mathbf{m}}\right)$ for a cylindrical geometry, in a way that has the equation (2):

$$
P_{m}(h)=\frac{2.42 E[h /(2 R)]^{5 / 2}}{\left(1-v^{2}\right)^{3 / 4}[L /(2 R)-0.45 \sqrt{h /(2 R)}]},
$$

Where $\mathbf{L}$ is the length of the cylinder in the case of a structure without frames, and $\mathbf{E}$ and $v$ are the Young's and Poisson's modulus of the material, respectively.

The maximum work pressure is given by the critical pressure $\left(\mathbf{P}_{\mathbf{c}}\right)$, displayed in the equation (3):

$$
P_{c}=\frac{\rho g H_{o c}}{\gamma}
$$

Where $\boldsymbol{\rho}$ and $\mathbf{g}$ are the density of water and gravity, respectively.

The maximum operational depth, Hoc, is estimated that may be around of $25(\mathrm{~m})$. In the calculations is considered, due to security restrictions, a safety factor of $\gamma=\mathbf{0 . 8}$, that it would increase the thickness of the shell and reduce the possibility of structure collapse.

To reduce the possibility of collapse according to different instabilities structural, the ABS proposes the following relationship, displayed in the equation (4).

$$
P_{c}=\left\{\begin{array}{ccc}
P_{m} / 2 & \text { si } & P_{m} / P_{y} \leq 1 \\
P_{Y}\left[1-P_{y} /\left(2 P_{m}\right)\right] & \text { si } & 1<P_{m} / P_{y}<3 \\
5 P_{y} / 6 & \text { si } & P_{m} / P_{y} \geq 3
\end{array}\right.
$$

Using equations (1), (2), (3) and (4), it's possible to calculate numerically the thickness of the shell, h, attending the different depths that it may have, from $0(\mathrm{~m})$ to $50(\mathrm{~m})$. 
In figure 2 it is showed the thickness that may have the pressure hull depending on the depth in the case of grade-A steel material.

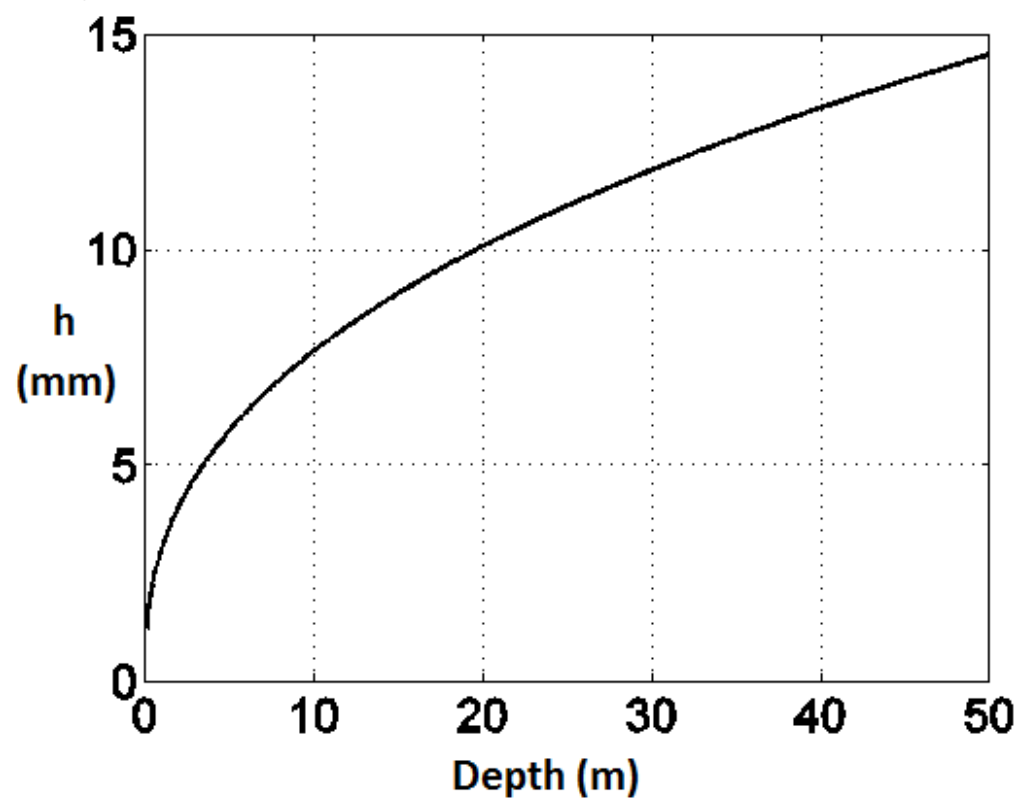

Fig. 2 Hull thickness vs depth

Figure 3 outlines the graphic of the thickness which the hull should have according to the yield strength and Young's modulus for different depths. In order the cylinder would be immersed to a greater depth, it is necessary to have a material with a higher yield strength and Young's modulus, and of this way to reduce the shell thickness of the hull and the total weight of the cylinder. In particular, if the cylinder is going down on the water to deep depths, it should be used a material with an elastic limit, $\sigma_{\mathbf{y}}$, higher as possible to reduce the thickness of the hull. On the other hand, to reduce the thickness of the hull to higher depths, the material should be with the higher Young's modulus possible.

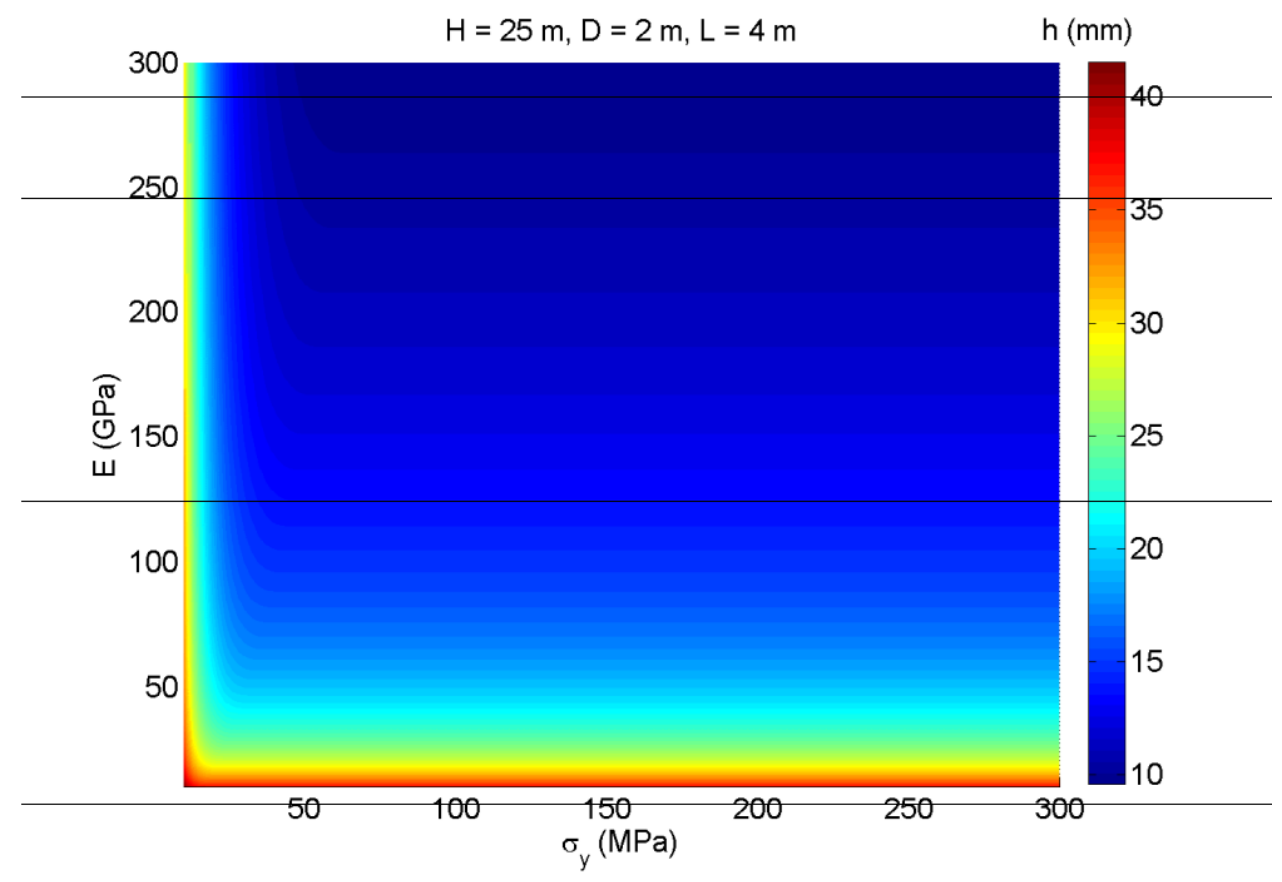

Fig. 3 Colourful graphics of the thickness value, h, which should have the hull based on the creep limit, $\sigma_{y}$, and Young's modulus, E, for the on-job cylinder depth 
Finally, an additional thickness has been considered in order to take in consideration the corrosion. Although a long-life painting process has been considered, it's important to consider the effect of the corrosion. After consider several references and sources in order to estimate the speed of the corrosion, it has been decided to take values from 0.13 (mm/year) to 0.2 (mm/year). Therefore, it has been estimated a conservatively corrosion suffered by an element of steel completely submerged in the sea.

If zinc anodes are used and also considering periodical reviews during its lifecycle of 20 years with cleaning works on the hull, coat renewals...the corrosion would not be more than one millimetre during all the useful life of the cylinder.

On the basis that the air system is communicated between all the hulls/floats, it's almost impossible that all the floats would flooding. If an accident occurs when the pressure inside is almost equal to the atmospheric, there is a big buoyancy that avoid the system goes down to the bottom.

If an accident occurs with all the void spaces and the system descends until the bottom, $50(\mathrm{~m})$, the pressure inside would be 2.3 (bar), which would allow decrease a few millimetres the thickness of the hull. This is really interesting not just for the cost but also for the weight.

As a summary, considering a corrosion of 0.2 (mm/year) for a lifecycle of 20 years and a depth of $20(\mathrm{~m})$, the thickness of the cylindrical plates of the hull must be $13.6(\mathrm{~mm})$. And if the submarine descends up to $50(\mathrm{~m})$, thickness should be about $15(\mathrm{~mm})$ without considering corrosion effects.

\section{Detail 3D model definition of the Double Pump Type Ballast Actuation System}

For the definition of the detail model of the DPTBAS, it is necessary to use Computer Aided Design Systems (CAD), which help engineers and designers in various industries, in the design and construction of aircraft, cars, bridges, digital cameras, ... and of course, also in ships and naval artefacts. There are other acronyms that often accompany the acronyms CAD, such as CAM and CAE. In both the first two letters mean the same, Computer Aided, and the third letter could be $\mathbf{M}$ for Manufacturing, or $\mathbf{E}$ for Engineering.

In the market there is a wide range of CAD systems, being the one used by us the FORAN ${ }^{\circledR}$ System, whose creation goes back to the 60 s of the last century [3].

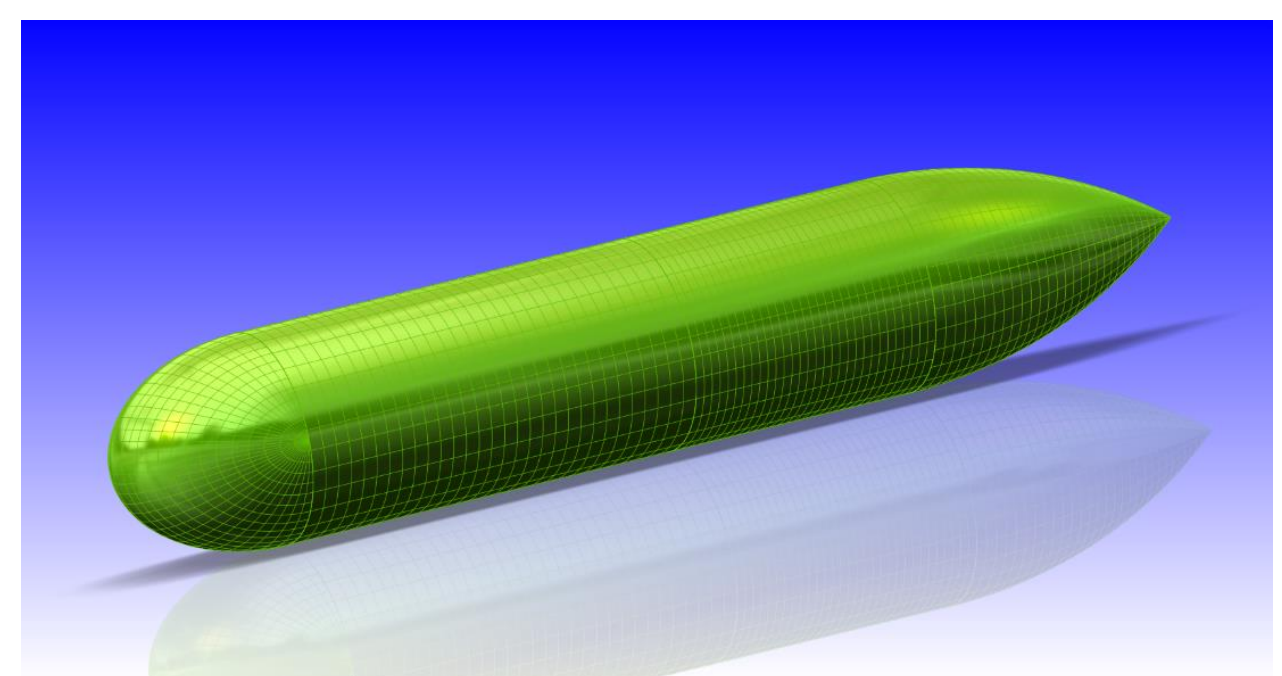

Fig. 4 Lateral hull shapes modelled with FORAN ${ }^{\circledR}$ System 
In order to design the DPTBAS, it is necessary to follow the process that follows with any other design of ship or naval artefact, which is governed by a series of stages. These stages are commonly divided, with the particularity of each geographical area, in Conceptual Design, Contractual Design, Basic Design, Detail Design, Manufacture and Maintenance.

One of the first tasks done in terms of design refers to the definition of shapes. Currently, most of the CAD Systems provide advanced tools for defining the surface model of the vessel based on the English acronym Non Uniform B-Splines (NURBS). Figure 4 shows the shapes of one of the helmets

The next step followed in the design of the DPTBAS was the definition of the layout, which included the design and management of all pump room spaces and the preliminary drawings of pumps, pipes, supports and accesses. Most of parts of this DPTBAS was compromised in the design phase, where the highest level of accuracy was required. During this stage different design alternatives could be studied.

The definition of the DPTBAS structure has been fully defined in a 3D environment. All work has been done on the ship's product model, which encompassed the basic design and detailing process in a single environment, which simplifies the organization and increases the overall performance of the design process. This solution meets the requirements of any shipyard or technical office through capabilities, automatic mechanisms and extensive use of topology and the existence of configurable procedures and macros. For example, in Figure 5, it is possible to observe the level of detail reached in the definition of the structure, where information has been added for manufacturing, such as chamfers, welded joints, accesses...

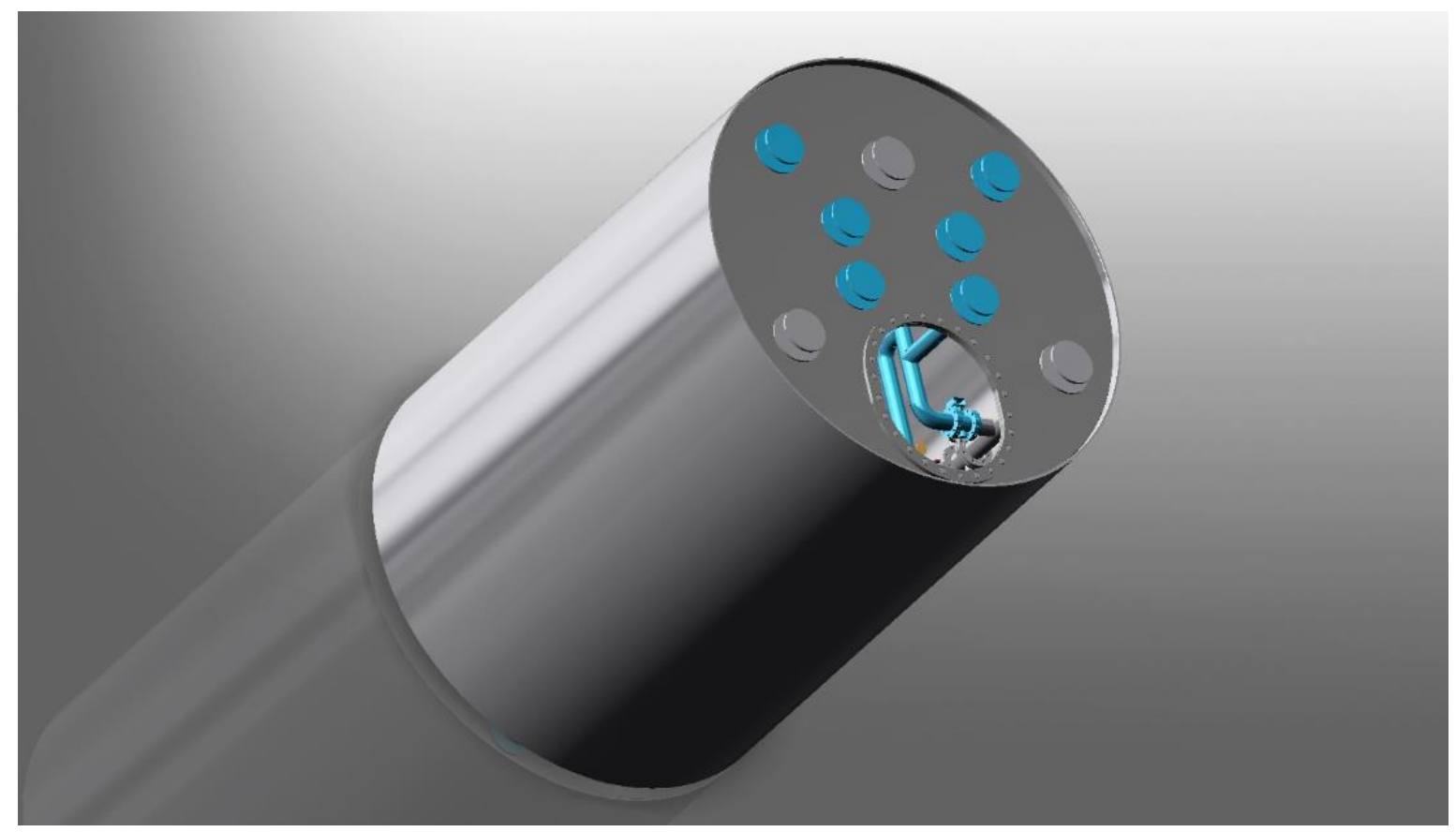

Fig. 5 Modelling of the structure of the DPTBAS, with information of chamfers for welding process

One of the most important characteristics of the use of these advanced systems of naval design is the integration of all disciplines. This property, combined with the continuity between the phases of basic design and detail, and together with the homogeneous development of all the modules, enables the realization of collaborative engineering in an effective way.

All information regarding standards, configuration parameters and other requirements of the shipyard is centralized in a database. The raw material catalogue, plate and reinforcement 
manufacturing methods and the parameters for configuring all shop drawings and outputs have also been defined.

An interactive 2D environment has also been used to define end types, holes, binders, ties, chamfers, and other parametric standards in which geometry is described from a macro of procedures that corresponds to a number of related elementary geometric operations.

The definition of standards has been created from another existing project. As a summary, the following three tasks have been performed in what has been standardized:

- Definition of the configuration parameters

- Creation of raw material

- Parametric standards.

As far as the definition of the main structure of the DPTBAS is concerned, curved and flat plates have been modelled in 3D, covering from the initial project to the detail. For this, two application contexts have been used, one for curved surfaces and another for flat ones.

It is important to emphasize that new concepts have been used for the initial design of the structure that have allowed a quick definition of a single 3D model that could be realized in the preliminary stages of the design. Even with a very preliminary definition of the hull surfaces, the level of detail increased with the definition of parts without assignment to a mounting unit or structural area. Topological relationships of ceilings, seams and curved plates have been used, incorporating advanced functions for a simple definition and modification of plates and holes.

The division of labour has been done using the concepts of surface, section and zone. Advanced splitting, multiple editing and multiple copying of the plates have been used, so attributes have been introduced in the detail design, with some of the capabilities that allow reuse of all information and significantly reduce the repetition of processes.

From the 3D model generated from the DPTBAS, it is possible to obtain workshop information for manufacturing and assembly and offer a more advanced way capable of generating, in an increasingly intuitive way, the information needed to build all the structural parts, obtaining reports and drawings.

The hull information introduced in the CAD system of the DPTBAS, for example, offers several methods for the expansion and bending of plates for the forming shop. You can create drawings and text files with all the relevant information, including numerical tables with the geometry of all the curves, for a manual marking or cutting, and in the database the pieces are stored for later nesting.

The drawings for the manufacture are automatically generated and include details of the end cut with automatic dimensioning, holes and notches, information about curving, attributes and the list of identical and symmetrical parts. It is also possible to define the assembly of curved panels with prefabrication beds. The base plane is automatically calculated and the projection of the panel in the base plane is shown for an interactive precision alignment of the panel on the guides. Configurable reports have been generated that describe all the relevant data for the assembly and marking of the panels.

An important part of the construction of the DPTBAS is the definition of its Building Strategy, therefore, we have established and organized the project according to the manufacturing and assembly processes that take place in the shipyard. The Building Strategy has been defined by organizing a hierarchical tree that describes the structure of the DPTBAS. The complete product model has been organized into a hierarchy of intermediate products and it has been possible to create alternative constructive strategies by providing interactive 
functions for the creation of intermediate products, the assignment of parts, the possibility of classifying interim products using configurable attributes for each type and definition of the assembly sequence, so that drawings and lists of intermediate products can be generated automatically.

Thanks to this, we are able to know the lengths and the classification of the type of welding according to different criteria (position and level of interim product), being able to obtain the information on welding through reports and plans.

Plates and profiles can be nested according to the shipyard's standards, production methods and manufacturing methods. It has been possible to calculate the raw material needed to produce the listing of materials and generate information for numerical control machines, drawings and cutting statistics during the detail design phase.

Regarding the DPTBAS outfitting part, the first thing is the definition of the standards. The following tasks were the first to be defined: libraries, representation groups, user attribute templates, pipe standards, classes and components of equipment and piping, acronym components of Heat, Ventilation and Air Conditioning (HVAC) such as libraries of auxiliary structures and supports.

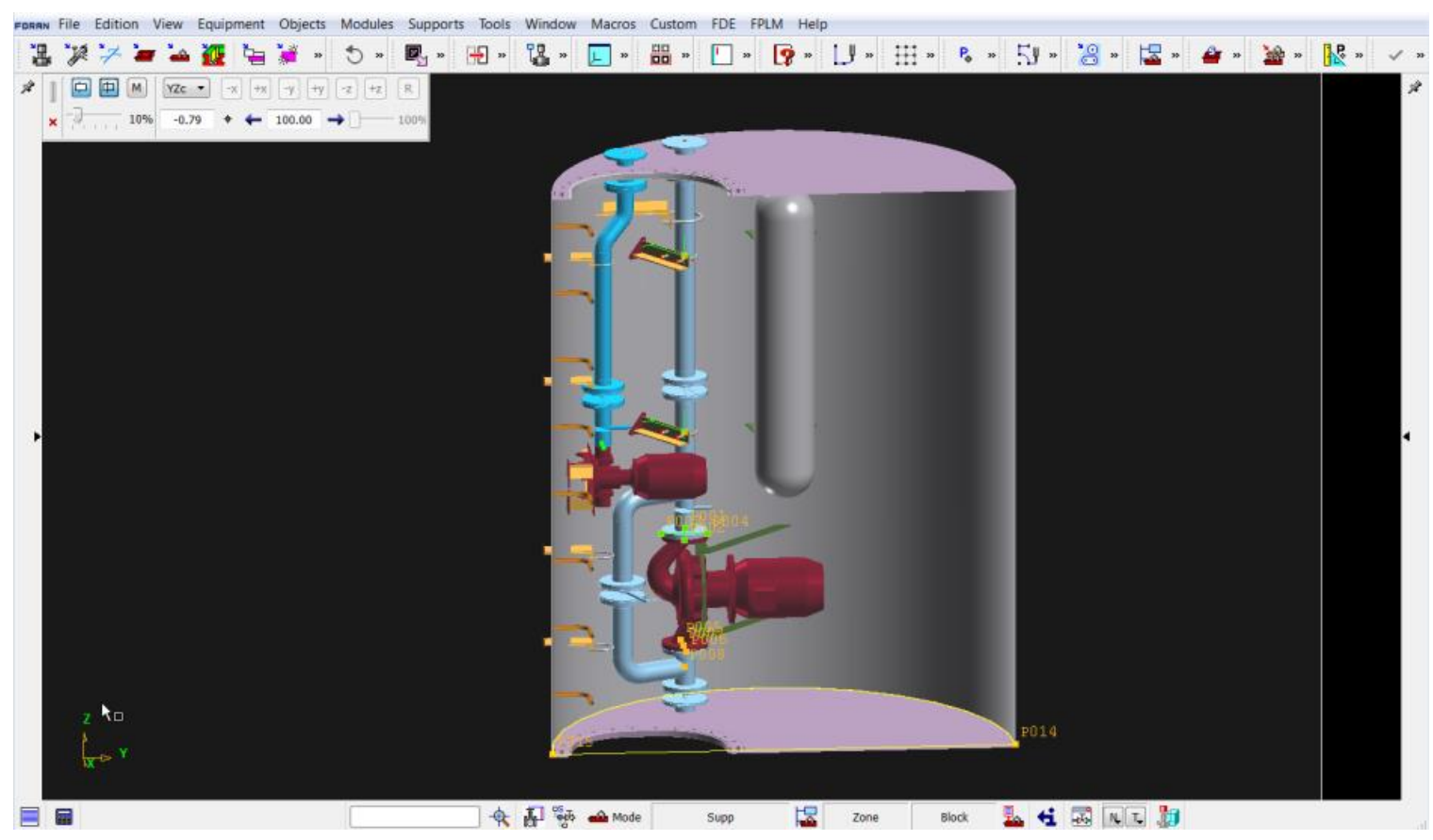

Fig. 6 Use of FORAN ${ }^{\circledR}$ System for intelligent pipe layout

The DPTBAS has been divided into zones (geographical) and systems (functional). In a 3D model environment the equipment and auxiliary elements have been defined (see figure 6). The 3D model objects have been assigned to representational groups in order to consider different levels of detail and include disassembly, security and operational representation areas. Technological attributes have been added to equipment model connections.

The equipment have been positioned, pipelines have been routed as well as the ventilation ducts and cable trays and insertion of accessories. The creation of auxiliary structures, arrangement of supports and the definition of spools of pipes and conduits have been completed.

Concepts for the initial design of outfitting have been inserted for a quick definition of equipment that can be used in the early stages of design, with direct access to existing 
information on the hull structure. It has been worked in a 3D environment, making a definition of the elements of the ship that allows reusing the information in the detail design.

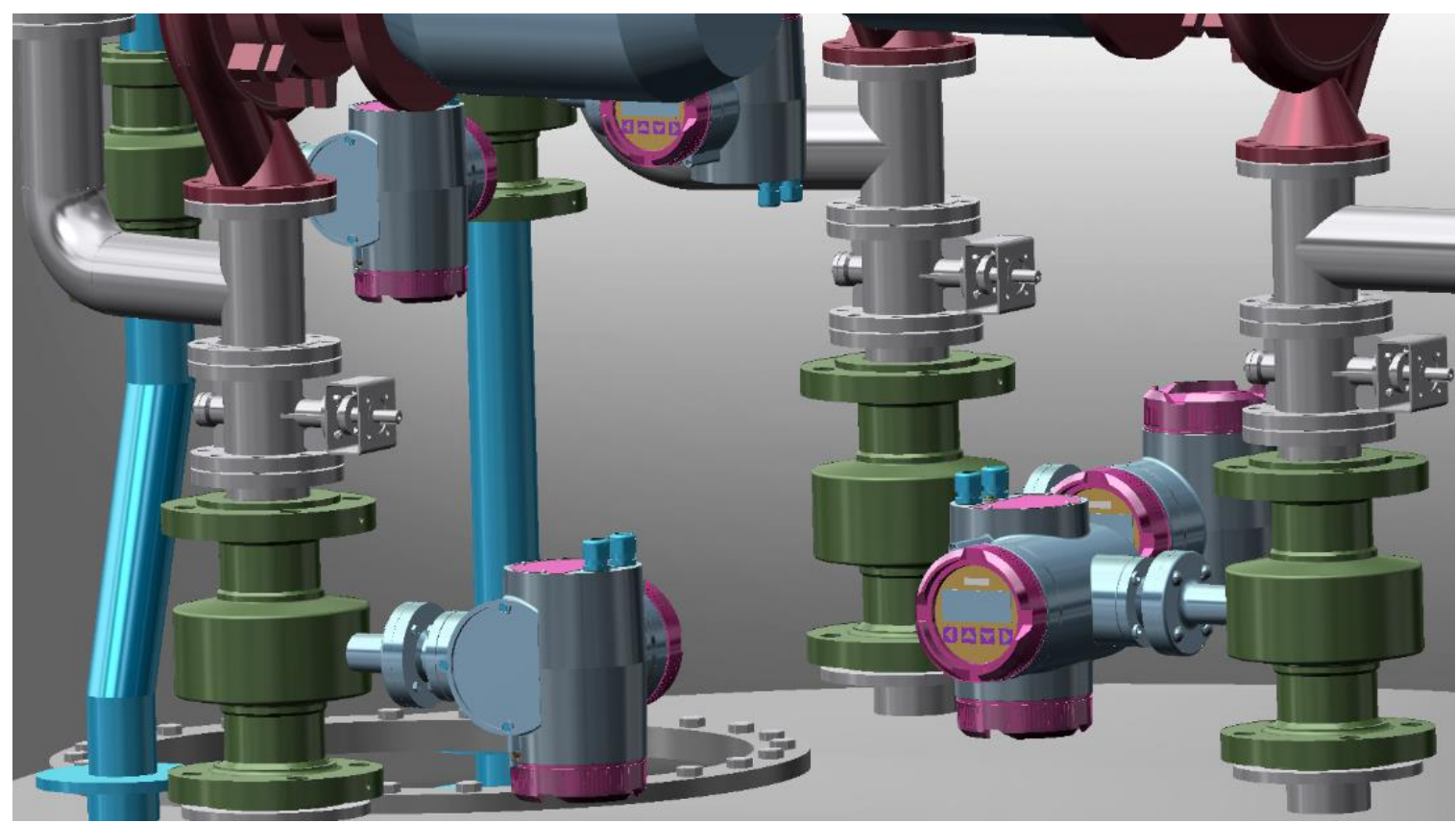

Fig. 7 Details of the position of the flow meters, where it is observed that the display is positioned optimally for consultation by the operators

All the available information on the hull structure elements, equipment, auxiliary structures, pipes and ducts has been introduced, as can be seen in figure 7 . The specifications of the pipes and the diagrams are integrated into the pipeline layout together with information on parametric fittings such as valves, flanges, branches, gaps, couplings, etc. Once the information is generated during the ship design process, it is possible to detect interferences and generate drawings and reports for the clashes.

Smart Pipe Routing tools have the properties of interactive pipeline layout, by defining an associated skeleton line or by arrangement of individual elements; The geometry of a pipeline may refer to the hull, decks, internal structure, equipment, auxiliary structure, ducts, trays, pipes or joints, or any significant point of an object; Automatic checking of pipe bending and identification of inconsistencies in the layout by the automatic consistency check between the Piping and Instrumentation Diagram (P\&ID) and the piping arrangement in the 3D model; As well as on-line interference testing.

Another element designed in the DPTBAS has been the auxiliary structures, such as ladders, which have been defined as a combination of a standard parametric plate and profile pieces. Each individual part has been managed like any other structural element for the purposes of producing and generating reports (for example for nesting). Auxiliary structures have been assigned to structure blocks and outfitting zones.

To create pipe supports and equipment foundations, the user defines it as part of the armament but is also associated with the structure blocks (such as plates and profiles). The supports are specific auxiliary structures linked to distributor type elements. A hierarchy is created to establish dependency between supports. Connectivity is formed between the supports and the supported elements, as can be seen in figure 8 . 


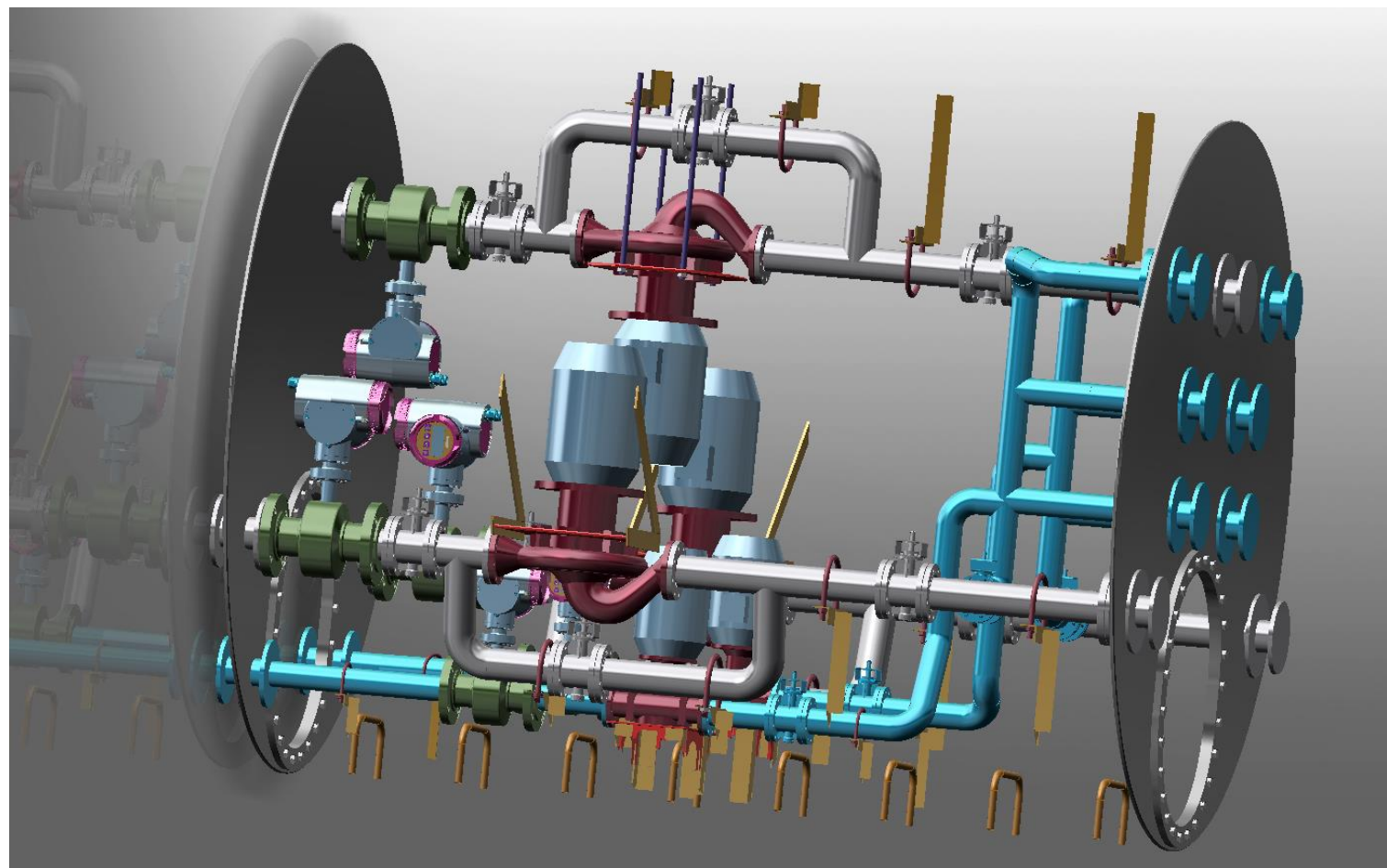

Fig. 8 Complete rendering of the Outfitting and piping systems of the Ballast System with supports, ladders and foundations

It is important to emphasize that before creating the $3 \mathrm{D}$ model, it is necessary to define the diagrams of the ship's systems, which in the case of the DPTBAS have been defined previously with this routing of distributors, pipe fittings with automatic control of connections, intelligent labelling of equipment, fittings and piping. There is an automatic association of graphic attributes depending on the fluid, the lists of materials are generated automatically and calculations are made in terms of pressure drop and flow, i.e. all the information created at this stage (equipment, piping and accessories) is available in the model 3D.

An especially important feature of the DPTBAS model is the automatic generation of isometric piping drawings according to the standards and formats of the shipyard where it is to be manufactured. Two types of isometric can be obtained: those designed for manufacturing in the workshop (known worldwide as spools): with all the information necessary for bending and the isometric ones for the assembly: with spools and accessories and providing the dimensioning relative to references of the DPTBAS.

The program in which the DPTBAS is modelled automatically controls the consistency between the pipes of the 3D model (figure 9) and the corresponding isometric drawings. Numerical control information could be exported to the bending machines of the yard's workshop. The pipe spools allow dividing the full pipelines into pieces of conformed pipes for manufacture and grouping them in isometric assemblies (manually or fully automatic). 


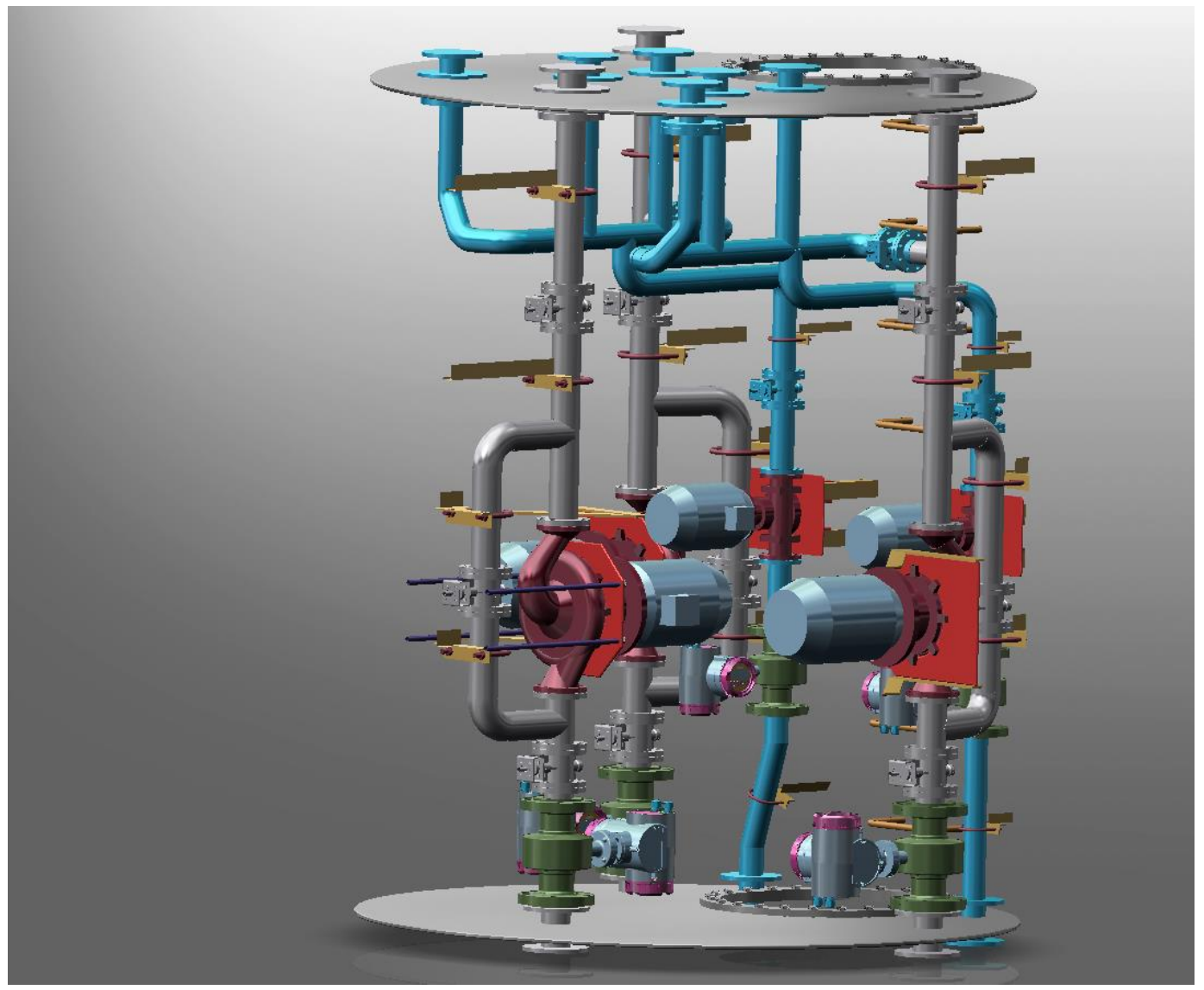

Fig. 9 3D Model of Ballast System, hiding the Compressed Air Service

Finally, it is possible to generate lists of materials related to equipment, accessories and pipelines, with the possibility of obtaining the information by services, zones or constructive strategy, such as:

- Lists of equipment, accessories and pipes by zones or services.

- Special symbol reports to / from the diagrams.

- Detailed list of materials.

- Pipe routing sequence.

- Situation of a particular element with respect to parameters previously defined as request of offer, reception of the order and assembly.

- Summary of accessories and pipes by zones or services.

\section{Conclusions}

The advantage of 3D piping routing provides the user with a more realistic experience of the final routing result of the DPTBAS.

Thanks to this, the designer can interactively traces the pipe segments by always attending to the surrounding elements, heights to be saved and possible complications that may arise.

It is essential to take into account the manoeuvrability and ergonomics when routing the pipe in the space available, for example in the pump room where space for crew work is essential. 
Routing in 3D allows the detection of interference with other objects in the environment such as equipment, auxiliary structures or other distributors, as well as elements of the structure of the ship resulting in the detection of collision and automatic execution of the intern or hole.

The realistic routing achieved in a 3D oriented CAD will allow to execute the calculations of pressure drop and minimum diameters with more reliability, always taking into account the elbows and heights in each routing between equipment, as well as the water hammer and the powers of connected equipment.

Obviously there are aspects that could be discuss further, as for example the ones regarding hull thickness, or the type of material that could be used. However, they could be part of a new research project.

\section{Acknowledgements}

This work has been partially supported by the formerly Spanish Ministry of Economy and Competitiveness under Research Grant DPI2014-53499R.

\section{REFERENCES}

[1] A. Lopez, J.A. Somolinos, L.R. Núñez, 2014. Patent about Dispositivo para el aprovechamiento de las corrientes marinas multi-rotor con estructura poligonal. Request number: P 201430182. Date: 11/02/2014. Granted by the OEPM 25/11/2014.

[2] R. Perez, A. Sanchez-Torres, 2015. Underwater exploration mission on Europa Jovian Moon. Internation Journal of Engineering Research and Science. Vol. 1. pp. 41-50.

[3] R. Perez, C. Gonzalez, 2015. Key CAD enhancements to fit submarine design requirements. ICCAS 2015. Royal Institution of Naval Architects. Bremen, Germany. 30 September and 1 August. Vol. 3. pp 153-163. ISBN 978-1-909024-43-4.

[4] A. Lopez, J.A. Somolinos, L.R. Núñez (2014). Energetic modelling of primary converters for marine renewable energies. Revista Iberoamericana de Automática e Informática industrial. Vol. 11. No. 2. pp 224-35.

[5] A. Lopez, et al, 2011. Dynamic Behaviour of a Second Generation Hydrokinetic Converter. IEEE Oceans Conference. Santander.

[6] L.R. Nuñez et al, 2011. The GESMEY Project. Design \& Development of a Second Generation TEC. 9th European Wave and Tidal Energy Conference (EWTEC'11), Southampton, United Kingdom.

[7] L.R. Nuñez et al. 2015. Comparative Analysis of Life Cycle Costs between the 2nd Generation TEC GESMEY and a 1st Generation TEC. 11th European Wave and Tidal Energy Conference. Nantes. EWTEC'15.

[8] J.A. Somolinos et al, 2012. Control de Profundidad de Cuerpos Sumergidos Basado en Cambios de Volumen. CEA. XXXIII Jornadas de Automática, Vigo, Spain.

[9] J.A. Somolinos, 2015. Control de operaciones de dispositivos marinos de aprovechamiento de la energía hidrocinética. Proyecto de I+D+I, del Programa Estatal de Investigación, Desarrollo e Innovación orientada a los RETOS de la SOCIEDAD. DPI2014-53499-R.

[10] J.A. Somolinos et al, 2016. PD Control with buoyancy compensation for automatic emersion maneuvers of first generation TEC. RENEW- Lisbon, Portugal.

[11] FORAN System. www.foran.es.

[12] L. Fernandez, E. Segura, M.P. Portilla, R. Morales, J.A. Somolinos, 2016. Dynamic Model and Nonlinear Control for a Two Degrees of Freedom First Generation Tidal Energy Converter. IFAC-PapersOnLine Vol.49. pp. 373-379.

[13] S. Desmeules, 2011, Stream flow hydroelectric generator system and methods of handling same, publication number: US13/112425. Date: 24/11/2011. 
Rodrigo Pérez, Amable López,

José A. Somolinos and Luis R. Núñez
Detail design of a Ballast Control Room for an Underwater Tidal Energy Converter
Submitted: $\quad$ 29.11.2016. Rodrigo Perez, Amable Lopez, José A. Somolinos y Luis R. Núñez Accepted: $\quad 02.10 .2017 . \quad$ (Spain)
Avd. Arco de la Victoria, No4, Ciudad Universitaria Madrid - 28040 